\title{
European radiographers' challenges from mammography education and clinical practice - an integrative review
}

\author{
Eija Metsälä ${ }^{1}$ - Nicole Richli Meystre ${ }^{2}$ - José Pires Jorge ${ }^{2}$ - Anja Henner ${ }^{3}$. \\ Tiina Kukkes ${ }^{4}$ Cláudia Sá dos Reis ${ }^{5,6}$
}

Received: 27 June 2016/Revised: 19 December 2016 / Accepted: 20 December 2016 /Published online: 16 March 2017

(C) The Author(s) 2017. This article is published with open access at Springerlink.com

\begin{abstract}
Objectives This study aims to identify European radiographers' challenges in clinical performance in mammography and the main areas of mammography that require more and better training.

Methods An extensive search was performed to identify relevant studies focused on clinical practice, education and training in mammography published between January 2010 and December 2015 in the English language. The data were analysed by using deductive thematic analysis.

Results A total of 27 full text articles were read, evaluating their quality. Sixteen articles out of 27 were finally selected for this integrative review. The main challenges of radiographers' mammography education/training can be divided into three groups: training needs, challenges related to radiographers, and challenges related to the organization of education. The most common challenges of clinical performance in mammography among European radiographers involved technical performance, the quality of practices, and patient-centeredness.
\end{abstract}

Eija Metsälä

eija.metsala@metropolia.fi

Nicole Richli Meystre

nicole.richli@hes-so.ch

José Pires Jorge

jose.jorge@hes-so.ch

Anja Henner

anja.henner@oamk.fi

Tiina Kukkes

tiinakukkes@nooruse.ee

Cláudia Sá dos Reis

claudia.sadosreis@curtin.edu.au
Conclusions The introduction of harmonized mammography guidelines across Europe may serve as an evidence-based tool to be implemented in practice and education. However, the variability in human and material resources as well as the different cultural contexts should be considered during this process.

Teaching Points

- Radiographers' awareness of their professional identity and enhancing multiprofessional cooperation in mammography.

- Radiographers' responsibilities regarding image quality (IQ) and optimal breast imaging performance.

- Patient-centred mammography services focusing on the psychosocial needs of the patient.

- Challenges: positioning, QC-testing, IQ-assessment, optimization of breast compression, communication, teamwork, and patient-centred care.

- Introduction of evidence-based guidelines in Europe to harmonize mammography practice and education.

Keywords Breast cancer · Mammography · Education . Clinical practice $\cdot$ Europe

1 Metropolia University of Applied Sciences, Mannerheimintie 172, PO BOX 4033, 00079 Metropolia, Finland

2 Haute Ecole de Santé Vaud, Av. de Beaumont 21, 1011 Lausanne, Switzerland

3 Oulu University of Applied Sciences, Kiviharjuntie 8, 90220 Oulu, Finland

4 Tartu Health Care College, Nooruse 5, 50411 Tartu, Estonia

5 Escola Superior de Tecnologia da Saúde de Lisboa, Av. D. João II, Lote 4.69.01, 1990-096 Lisboa, Portugal

6 Department of Medical Radiation Science, Curtin University, GPO Box U1987, Perth, WA 6845, Australia 


\section{Introduction}

Mammography remains the imaging modality that has proved its cost-effectiveness for screening and diagnosis of breast diseases $[1,2]$. For that reason, it is one of the X-ray examinations most frequently performed for the healthy female population in the world. Due to the radiation, mammography requires optimized practice [3]. In order to ensure this, quality standards must be implemented and followed. These standards should address all aspects in the mammography examination, including equipment, performance, provider profile, and healthcare staff. Monitoring the quality standards provides opportunities to improve practice through a feedback mechanism that allows the identification of sub-optimal practice, and can suggest difficulties, training needs, and challenges [4-10].

Radiographers face challenges in clinical practice due to upgrades in technology, equipment variability, the introduction of new techniques, the culture of each healthcare institution, patient interaction, and the requirements for continuous professional development $[11,12]$. To follow all updates and to be aware of all requirements, education and training are crucial, although the attendance can be affected by costs, work constraints, geographical location, timing, quality issues, family constraints, a perceived lack of benefits, and a lack of relevance, support and availability of education and training regarding the several needs [11].

The quality of breast cancer screening can be defined by using several quality outcomes. The European Guidelines for quality assurance in breast cancer screening and diagnosis mention 39 key performance indicators for mammography screening [13]. The quality of breast cancer screening can roughly be divided into the (screening) process quality, the quality of practices, and technical quality. The quality of the screening process comprises the invitation to the screening programme, the justification and optimization of mammography examinations, the staff training and qualification requirements, recording and reporting examination data, as well as the attendance rates $[14,15]$. Patients' wellbeing and health is the ultimate goal of any screening programme. Patient-centeredness and especially patient-provider communication is known to be associated with attendance rates of screening programmes [16]. This aspect is also central in the European Guidelines for quality assurance in breast cancer screening and diagnosis [13]. The quality of practices comprises the determination of patient doses and their comparison with the diagnostic reference levels, clinical image quality assessment, self-assessment, and clinical audits. Technical quality assurance consists of acceptance testing and quality control during the use of equipment. This quality dimension considers image acquisition, image processing, and imaging display [14, 15]. Due to the emphasis on patient-centeredness as an important component of screening process quality, it was chosen as the framework for deductive thematic analysis in order to widely cover the aspects of breast cancer screening quality. In this integrative review, the quality of breast imaging service was also considered from the viewpoints of staff education (radiographers), technical quality, and the quality of practices.

The aim of this study was to identify European radiographers' challenges in clinical performance in mammography and the main areas of mammography that require more and/or improved training.

The following search questions were set:

1. What are the most common challenges of mammography training for radiographers?

2. What are the biggest challenges for radiographers from the viewpoints of (1) technical performance, (2) quality of practices, and (3) patient-centeredness in the breast imaging service?

\section{Materials and methods}

An extensive search was performed to identify relevant studies focused on clinical practice as well as on education and training in mammography published in the English language between January 2010 and December 2015. The Population Intervention Context Outcomes (PICO) strategy (Table 1) was used for the construction of research questions and for the bibliographical search. It is reported to facilitate the definition of population groups, interventions, comparators, and outcomes of interest. It was also used as support to define the inclusion and exclusion criteria. For the comparison (C), it is possible to replace it with the identification of context (Co) which is relevant, especially in (non-comparative) qualitative studies [17].

Table 1 The PICOs in search questions 1 and 2

\begin{tabular}{|c|c|}
\hline The PICO for question 1 & The PICO for question 2 \\
\hline Population: radiographer & Population: radiographer \\
\hline $\begin{array}{l}\text { Intervention: mammography } \\
\text { education }\end{array}$ & $\begin{array}{l}\text { Intervention: breast imaging } \\
\text { service }\end{array}$ \\
\hline Context: & Context: \\
\hline Outcomes of Interest: challenges & $\begin{array}{l}\text { Outcomes of Interest: challenges } \\
\quad \text { of } \\
\text { technical performance } \\
\text { quality of practices } \\
\text { patient-centred services }\end{array}$ \\
\hline
\end{tabular}




\section{Evidence review strategy}

The integrative review method that allows combining studies with a variety of research designs was used [18]. The following electronic search engines and databases were used: EBSCO Host: Academic Search Elite; CINAHL with Full Text; CINHAL, and Science Direct. We also searched Pro Quest: ABI/INFORM Complete; Applied Social Sciences Index and Abstracts (ASSIA); Biological Sciences; British Humanities Index (BHI); ERIC and MEDLINE and the Open Access Theses and Dissertations database (OATD). According to authors' previous experience in database search in the field, the most relevant findings can be found by using keywords and their combinations with the command that these must either be found in the title or abstract of the article. The subject headings and keywords searched were teaching OR learning OR education; radiographer (with related/allied search option) OR radiologic technologist; mammography OR breast screening; challenge; quality OR image quality; patient-centred; and evaluation. These subject headings and keywords were used in similar combinations in the several databases, and with the option that these must either be found in the title or abstract of the article if that was possible in the particular database.

Inclusion criteria for the selected studies were the focus on European radiographers' work in breast screening or clinical mammography, population based or opportunistic screening, describing the challenges of the breast imaging service or education, technical aspects, quality of practice, and the patient-centred viewpoints on mammography. We included qualitative and quantitative peer-reviewed studies, intervention studies, and research and development projects with sound methodology comprising the JBI levels of evidence for effectiveness from 1 to 3 and the levels of evidence for meaningfulness from 1 to 3 [19]. The search was limited to papers published in 2010 or later since the studies published before that year may not be relevant as the imaging technology and quality assurance practices are developing fast.

The search was performed in the databases between 1 October and 1 November 2015, looking through all the titles and selecting the relevant titles for the abstract search. Two reviewers then studied the abstracts and the full texts independently. The relevant articles were chosen within the full text review based on a consensus discussion. The agreement percentage was $81 \%$ (22/27) before the consensus discussion and $100 \%$ after the discussion. The reviewers evaluated the quality of the reporting in the studies independently according to the modified version of the STROBE v4 checklist for cohort, case-control and cross-sectional studies (combined) [20]. This type of evaluation criteria has previously been used in several published integrative reviews [21-23]. Detailed evaluation of the quality of each study's methodology and conduct was not performed because the focus of the review was rather to identify the dimensions than the effect of the phenomenon. It aimed to identify challenges in training and practice in the field. The studies that did not achieve the JBI levels of evidence for effectiveness $1-3$ and the levels of evidence for meaningfulness 1-3 were excluded. Also, the studies receiving two or more 'hardly or not at all satisfies assessment criteria' scores in the STROBE checklists were rejected. However, these evaluation methods were consistent, i.e., a study that got two or more 'hardly or not at all satisfies assessment criteria' scores, rarely reached the JBI level 3. In case of a mismatch in quality evaluations, a consensus was discussed (Table 2).

The shortened version of the STROBE checklist was used due to the need to include studies with several types of designs

Table 2 Critical assessment of the reporting of the studies

Ref. Assessment criteria of the studies.

\begin{tabular}{|c|c|c|c|c|c|c|c|c|c|}
\hline 1. & 2. & 3. & 4. & 5. & 6. & 7. & 8. & 9. & 10 \\
\hline$* *$ & $*$ & $* *$ & $* *$ & $*$ & $*$ & $* *$ & $* *$ & - & $* *$ \\
\hline$*$ & $* *$ & $* *$ & $* *$ & $*$ & $*$ & $*$ & $*$ & $* *$ & $* *$ \\
\hline$* *$ & $*$ & $* *$ & $* *$ & $* *$ & $*$ & $*$ & $* *$ & $\mathrm{X}$ & $* *$ \\
\hline$*$ & $*$ & $*$ & $* *$ & $*$ & $* *$ & $* *$ & $* *$ & $*$ & $* *$ \\
\hline$* *$ & $*$ & $* *$ & $* *$ & $*$ & $*$ & $*$ & $* *$ & $*$ & $* *$ \\
\hline$* *$ & $* *$ & $* *$ & $*$ & $*$ & $* *$ & $*$ & $* *$ & $* *$ & $* *$ \\
\hline$* *$ & $*$ & $* *$ & $*$ & $* *$ & $* *$ & $*$ & $* *$ & $*$ & $*$ \\
\hline$* *$ & $*$ & $* *$ & $*$ & $* *$ & $*$ & - & $* *$ & $*$ & $* *$ \\
\hline$* *$ & $* *$ & $* *$ & $* *$ & $* *$ & $* *$ & $*$ & $* *$ & $*$ & $* *$ \\
\hline$* *$ & $*$ & $* *$ & $* *$ & $* *$ & $* *$ & $* *$ & $* *$ & $*$ & $* *$ \\
\hline$* *$ & $*$ & $* *$ & $* *$ & $*$ & $* *$ & $*$ & $* *$ & $* *$ & $* *$ \\
\hline$* *$ & $*$ & $* *$ & $* *$ & $*$ & $* *$ & $* *$ & $*$ & $*$ & $* *$ \\
\hline$* *$ & $*$ & $* *$ & $*$ & $*$ & $*$ & $*$ & $* *$ & $*$ & $* *$ \\
\hline$* *$ & $* *$ & $* *$ & $* *$ & $*$ & $* *$ & $* *$ & $* *$ & $*$ & $* *$ \\
\hline$* *$ & $* *$ & $* *$ & $* *$ & $\mathrm{X}$ & $* *$ & $\mathrm{x}$ & $* *$ & $*$ & $*$ \\
\hline$* *$ & $*$ & $* *$ & $*$ & $*$ & $* *$ & $*$ & $* *$ & - & $*$ \\
\hline
\end{tabular}

1. Study background and theoretical framework are clearly defined.

2. Purpose, aim and research questions are clearly defined.

3. The design is clearly stated.

4. The setting is clearly described.

5. For independent and dependent variables, confounders are clearly identified and consistently implemented or something else should be added here.

6. Data sources and analysis methods are clearly described.

7. Efforts to address potential sources of bias are described.

8. Research questions are answered logically.

9. Study limitations and generalizability are discussed.

10. Relevance to the topic.

** assessment criteria are satisfied

* assessment criteria are partly satisfied

_ assessment criteria are hardly or not at all satisfied

$\mathrm{x}$ assessment criteria do not apply. 
into this integrative review. In addition, the use of this strategy allows commensurable evaluation of all the studies, showing the evaluation results in a table format. The results of the selected studies were analysed using deductive thematic analysis, a suitable option because there were studies with different kinds of designs. The challenges of European breast imaging practices were categorized under three dimensions of breast imaging quality: a) technical performance, b) quality of practices, and c) patient-centred viewpoints. The first two dimensions have been defined in the theoretical background of this study $[13,14]$. The third dimension, also mentioned in our PICO components, was clearly separate, stemming from the studies forming the data of this integrative review.

\section{Results}

With the selected keywords and their combinations, a total of 299 results were obtained using the following databases and search engines: Medline - 96, Pro Quest - 68, Science Direct 37, EBSCO Host - 58, and OATD (theses and dissertations database) - 38. Two relevant titles were found by hand search. The most typical reason for exclusion was the lack of relevance for the search questions. Abstracts of 48 articles were read, and 21 were excluded after their evaluation. Reasons for exclusion at the abstract level were associated with the issues of relevance, the type of the article or the target group. A total of 27 full text articles were read to evaluate their quality. Sixteen articles out of 27 were finally selected for this integrative review (Fig. 1). Reasons for rejection at the evaluation of full text level involved a low reporting quality not satisfying the evaluation criteria mentioned in Table 2: one article was rejected based on criteria 1 and 8 , two based on criteria 7 and 10 , and also due to the focus on the technical performance of devices only or patient satisfaction only. One article was rejected based on criteria 3 to 6 and also because the target group was not radiographers $(n=1)$. One article, a case-control study not performed in Europe, was rejected because of JBI levels.

\section{Description of the selected studies}

Seven out of the 16 selected studies had been performed in the United Kingdom, three in the Netherlands, two in Portugal, two in Serbia and/or Croatia, one in Turkey, one in Norway, and one involving several countries in Eastern Europe. Most of the studies were quantitative $(n=12)$, three studies were qualitative, and one study used a mixed method approach. In five out of the 16 selected studies, the equipment in use was screen-film mammography (SFM) [24] or SFM combined with digital mammography (DM) [5, 25-27]. Four of these research settings using SFM systems were located in Eastern Europe [5, 25, 26] (Table 3).
The focus of six selected studies was on the compression force and its association with dose [27-31]. Four studies evaluated image quality in mammography at national levels [5, 25, $27,31]$, two studies at local levels [26, 32], and one study about image quality evaluation was multinational [24]. Three studies considered radiographer training related to mammography $[31,33,34]$. There was also a study focusing on the evaluation of clinical image quality [32], especially on positioning [35], and one study focusing on patient viewpoints of mammography examination [36] (Table 3).

\section{Challenges in mammography education}

All the studies addressed radiographer training (to a greater or lesser extent), but all of them identified the need for additional training in a wide range of issues or contents in order to achieve high quality in mammography services.

Thematic analysis of the selected study results produced three main challenges of mammography training for radiographers: (1) training needs, (2) radiographer related challenges, and (3) challenges related to the organization of education. Training needs comprised four subthemes, each of them representing a specific area of training needing to be developed in mammography education: (1) multiprofessional cooperation within the diagnostic process of breast cancer (BC) [5]; (1) image quality such as artefact recognition, quality control and dosimetry as well as implementing and maintaining quality control and quality assurance $[5,24,25,31$, 32]; (3) competences in patient-centred work such as counselling patients in the issues related to mammography or taking into account the psychosocial needs and comfort of the patient [25], and (4) performance of breast imaging in an optimal way, including breast pathology, and performing basic digital mammography and further examinations, e.g., interventional procedures of the breast tissue and tomosynthesis [31]. Radiographer related challenges mentioned in the selected studies included a lack of commitment and motivation, and the effective delivery of the training, particularly in relation to role extension associated with the amount of time required for radiographers to learn and rehearse the script [33]. Challenges related to the organization of education involved information about the education and giving feedback to those who attend these trainings [33] (Table 3).

\section{Challenges of mammography practice}

The second PICO question based on deductive thematic analysis, Challenges of the breast imaging service, focused on technical performance, quality of practices, and patient-centeredness. Considering the challenges of technical performance, the following ten aspects were found: cleaning and artefacts [5, 24, 26], AEC [5, 24], the performance of periodic tests $[5,26]$, exposure parameters $[5,31]$, receptor 
Fig. 1 The selection process of the studies

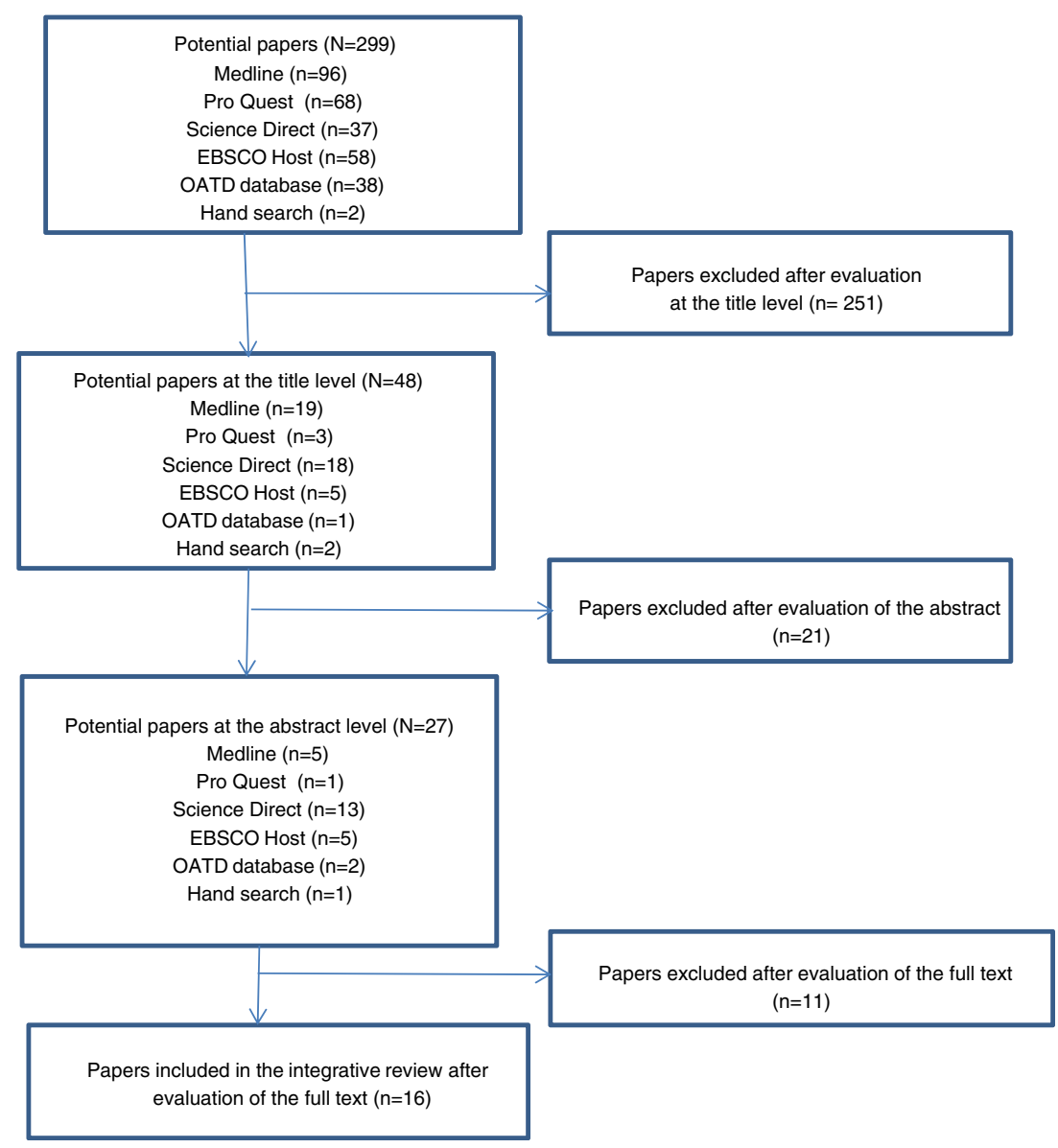

handling [5, 24, 31], screen-film combination [24], viewing conditions [24, 25], breast compression, interprofessional working [24, 25, 27-31, 37, 38], the use of positioning sheets [35], and the implementation of quality control (QC) programs [24, 26, 31] (Table 4).

In the selected studies, there were nine types of challenges associated with the quality of practices in mammography, comprising the following areas: positioning especially in MLO projection [25, 26, 31, 35]; image contrast [5]; artefacts [25]; the variations in image quality using screen-film, CR and DR systems [33]; the implementation of dose reference levels (DRL) [34]; (a lack of) the implementation of repeat/reject analysis [31]; image processing [26]; image labelling and documentation [25]; and the variability of strategies for image quality evaluation [32] (Table 4).

Challenges of mammography practice associated with the patients comprised: (1) the provision of seamless and multiprofessional diagnostic services emphasizing the importance of staff skills and attitude to the quality of breast screening experience [36], (2) a lack of the possibility for the staff to use enough time with the patient due to the heavy workload [31], (3) promoting breast screening adherence in radiographer's work [33], and (4) the use of compression force [29, 30, 35] (Table 4).

\section{Discussion}

\section{Challenges of mammography education}

According to the European guidelines for quality assurance in breast cancer screening and diagnosis [13, 19], a breast unit must have a core team composed of health professionals of various disciplines who have undergone specialist training in breast cancer beyond that given in their general training. This emphasizes the importance of training in multiprofessional cooperation amongst radiology staff, and the same result was also found in this integrative review [5]. Communication and social skills are also mentioned among the central competences of radiographers in these guidelines $[13,19]$. In this integrative review, it was evident that radiographers lacked competence for working in a patientcentred way $[25,36]$.

According to the selected studies, it is possible to verify the evidence related to the co-existence of both obsolete (SFM) and modern technology (DM) in European countries. This can have an impact on practice and also on radiographer education and training in mammography. The levels of knowledge and the topics to be addressed need to be country specific, promoting harmonization in both areas (clinical practice and 


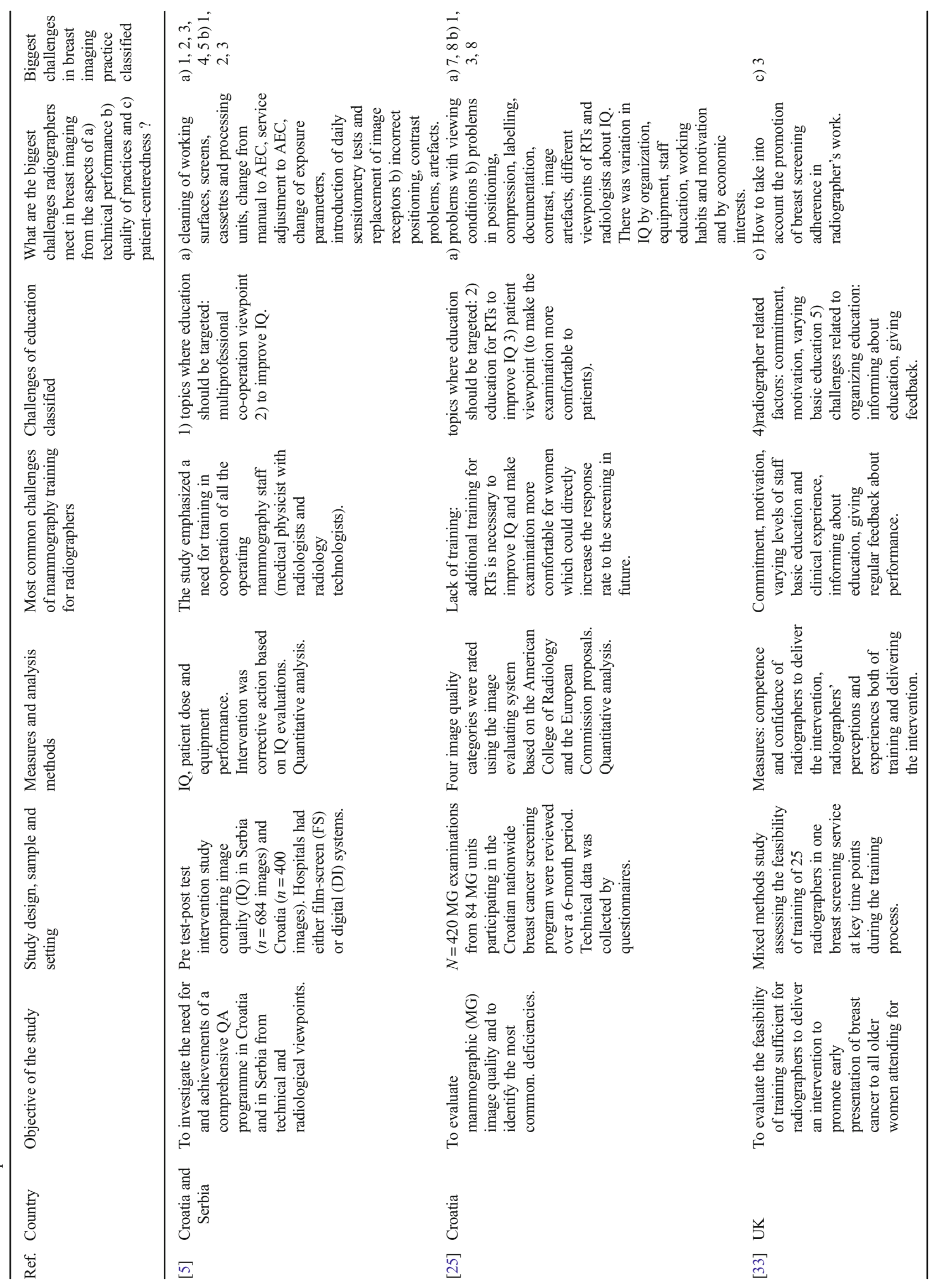




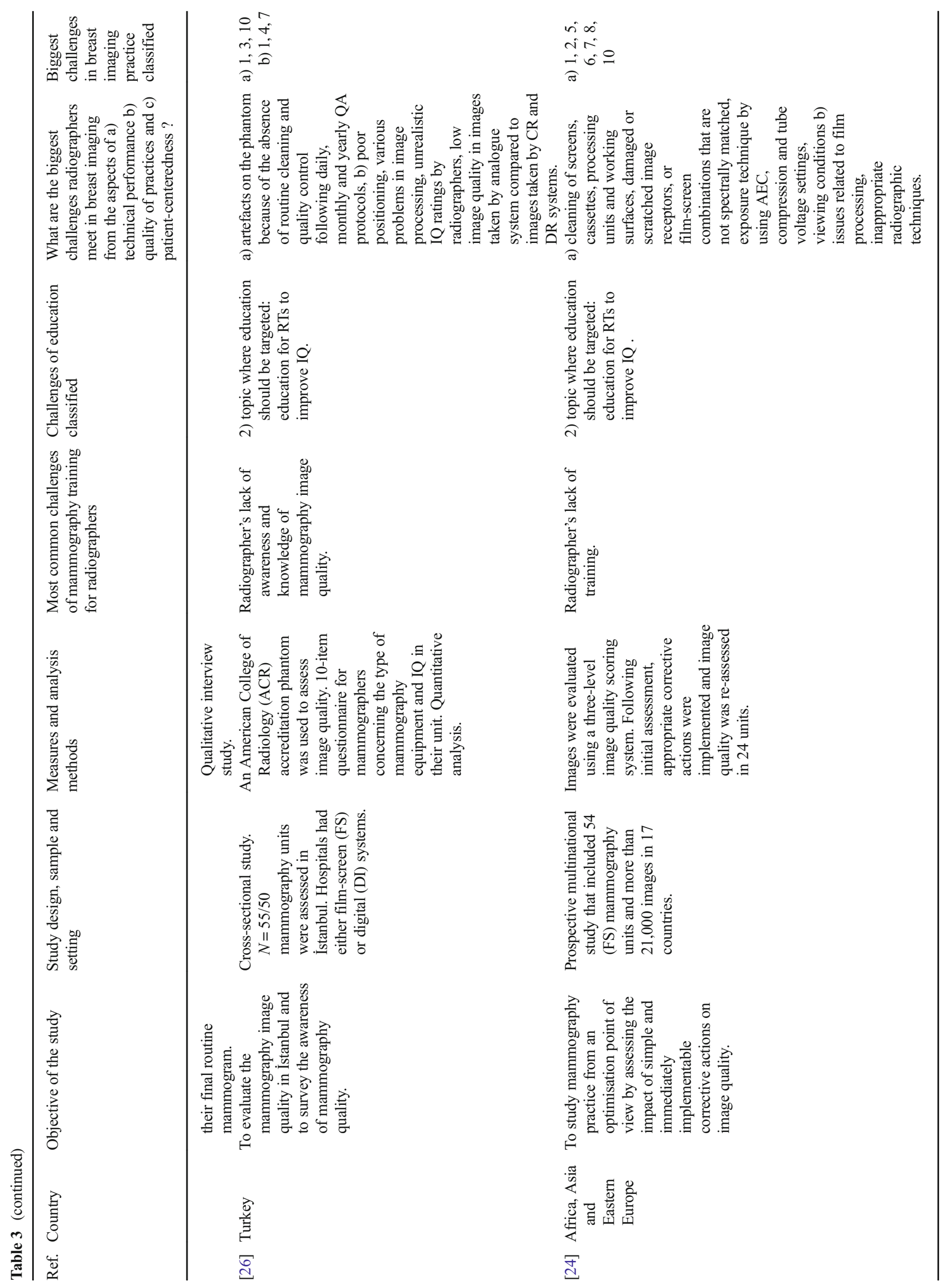




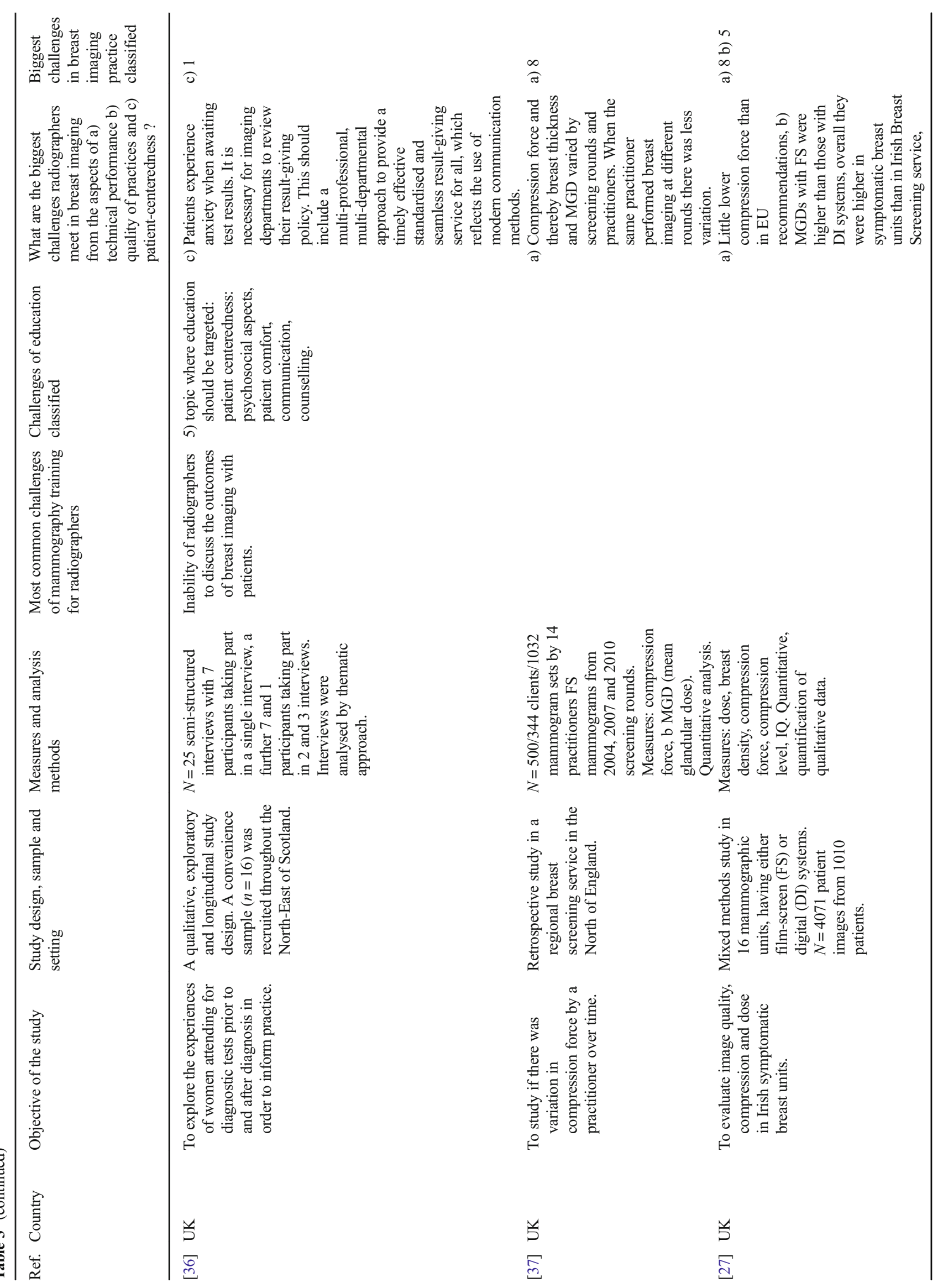




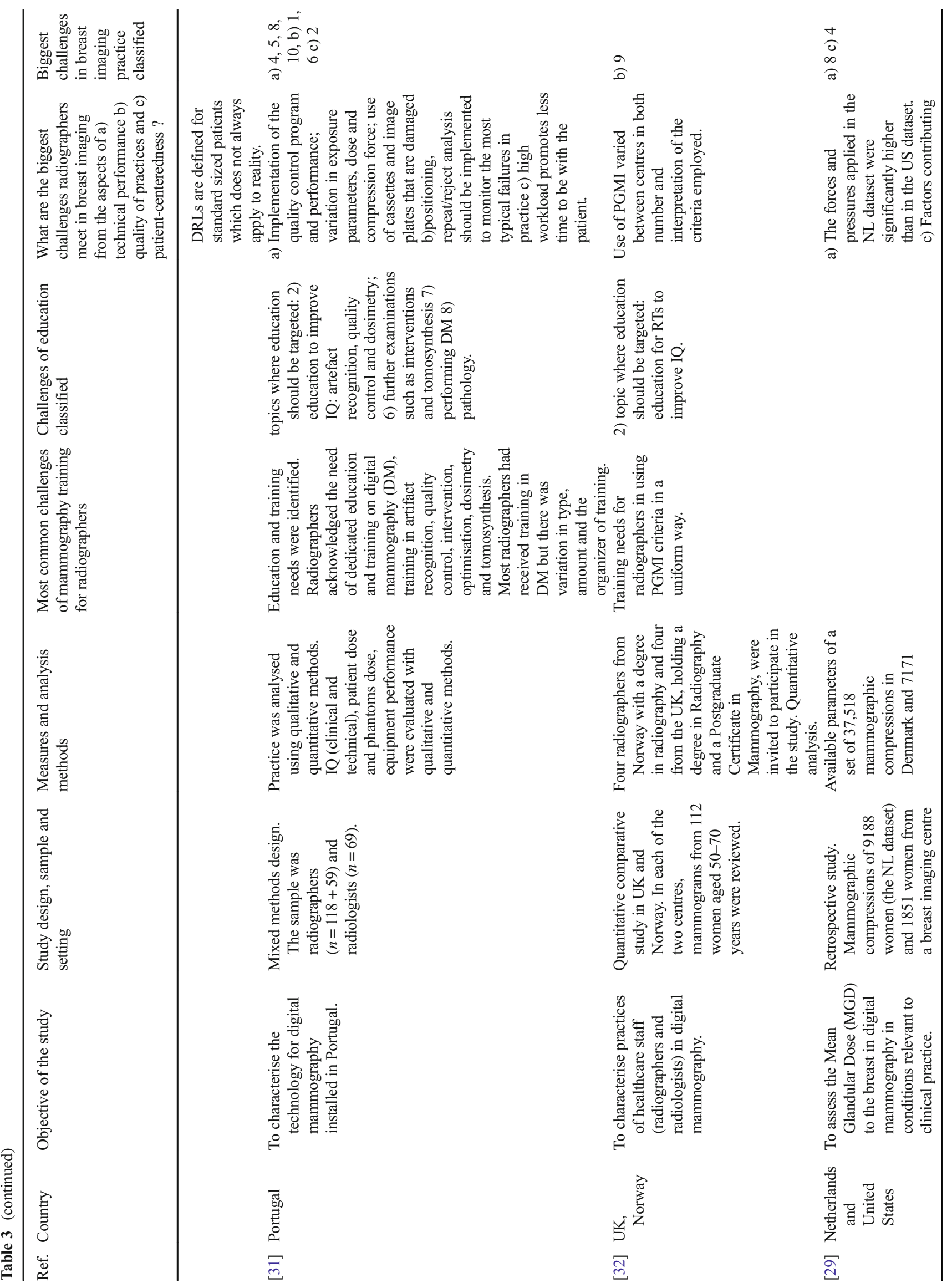




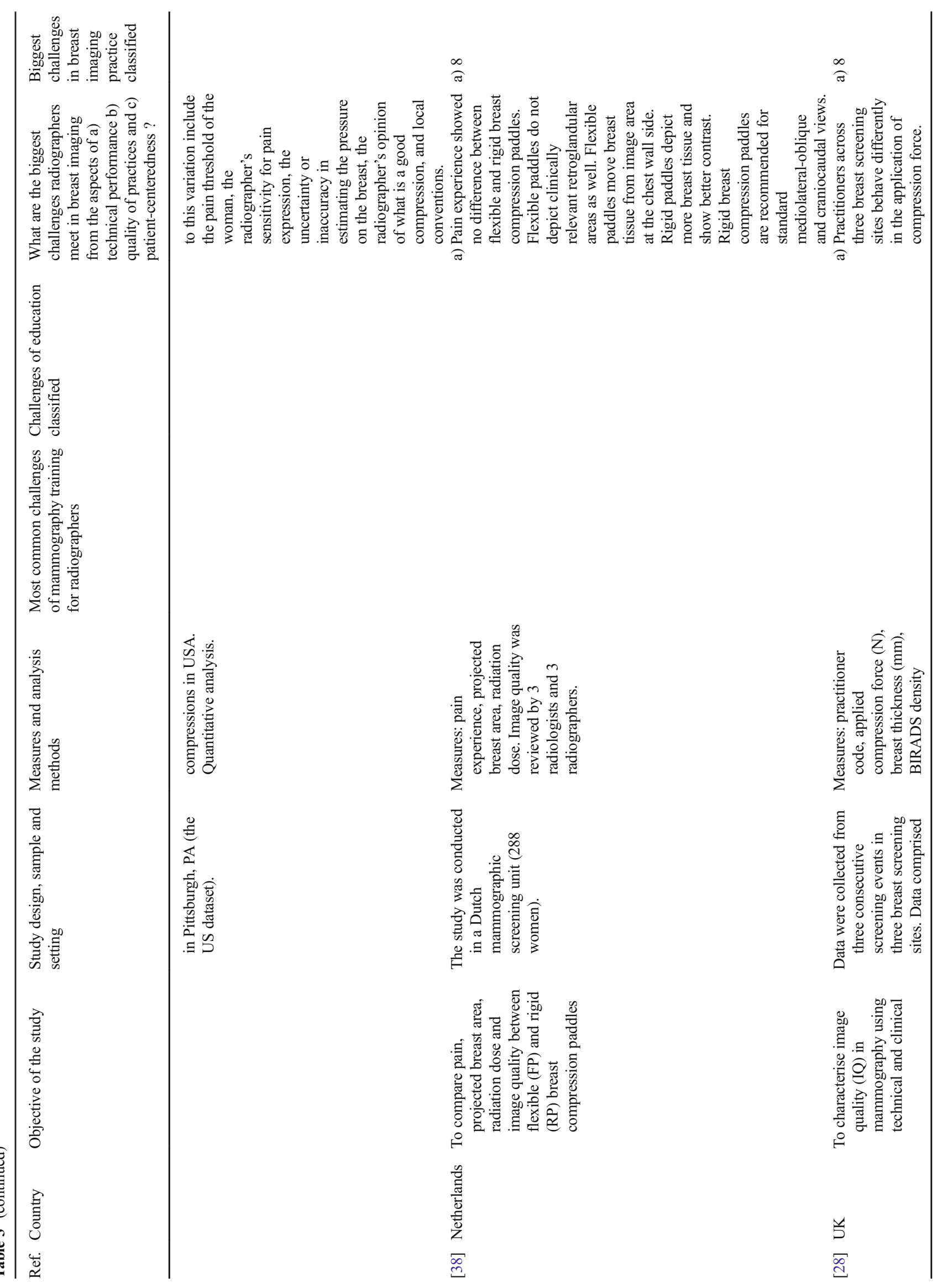







training). The training of the students and radiographers should meet the actual needs in their country.

Despite different contexts and equipment, some challenges of education and practice can coexist in several countries, being more related to the lack of motivation and commitment [33]. That was also observed in a previous study related to the continuous professional development of Portuguese radiographers. In that study it was also verified that the area of mammography was less valued in terms of education and training compared to other imaging modalities [39], which may compromise the improvements in this field. Another aspect that has an impact on the quality of mammography services is the heavy workload [31] that may also have an impact on student and educator motivation due to work demands and the lack of time, making education also a challenge under these circumstances.

The analysed studies also showed a concern about basic radiographer training and its sufficiency in order to develop proper technical skills, including accurate positioning, quality control, dosimetry, and teaching skills. The difficulty for our review is that the training differs from one country to another and that the papers do not describe the performance of training in the countries where the studies were performed [5, 24, 25, 31, 32.] Moreover, the educational needs of experienced mammography radiographers must be differentiated from the training needs of the radiographers only starting their work in the breast cancer screening programme. However, the EUREF guidelines and the other European guidelines [31,32] recommend that radiographers should attend 40 hours of continuous professional development (CPD) training per year, ranging from two to six weeks, depending on the individual performance and experience [31], to improve their basic skills and to stay up to date. Nevertheless, no evidence was found in the selected studies about how many countries follow the European recommendations rigorously. In order to ensure good attendance rates to CPD type of mammography education, it should be made mandatory in all European countries, information about the education opportunities should be properly communicated, and attention should be paid to the pedagogic and technical organization of the education [33].

\section{Challenges of mammography practice}

In this article the challenges of breast imaging practice are divided into three areas: technical performance, quality of practices, and the patient-centred way of working. The challenges related to technical performance and quality of practices like the issues of positioning, breast compression, contrast, noise, artefacts, sharpness, and labelling, have been known for a long time, and identified also in the studies performed outside the European context [26, 32-34, 40-43]. The introduction of digital mammography in the practice brought challenges in breast positioning, mainly due to a bigger breast 
support according to radiographers [32]. Small breasts and shorter clients are difficult to position without the superimposition of the arm and/or the abdominal wall.

According to Whelehan et al. [44], clients avoid reattending for screening mammography due to breast pain experienced in the previous exams. During a mammography exam, pain can arise from the application of compression force, being also a challenge in the radiographer practice, as the radiographer rather than the client influences the application of compression force in mammography. The application of compression force and the resultant pain can affect client experience, radiation dose and image quality (noise, sharpness, movement artefacts), and needs to be addressed in radiographer education and training. Mercer et al. [28] also highlighted in their study the considerable variability in breast compression force applied during mammography examination, showing room for improvements in practice and consequently in radiographers' education. It is crucial to identify strategies allowing the optimisation of breast compression, resulting in pain reduction and improving the image quality.

Technical challenges of the AEC implementation are also common and well known regardless of the cultural context [45]. Other challenges of technical performance that were identified in these European studies involved the performance of periodic quality control tests and the use of exposure parameters and quality control programs in general (Table 4). When the AEC systems are not well calibrated, the selected exposure parameters are affected. Image quality can be insufficient with higher levels of noise, complicating diagnosis, and/or the breast dose can be higher than necessary, affecting the radiation protection of the patient (the dose creep phenomenon). The dose creep can also promote a loss in image quality due to overexposure [31]. However, improvement in quality has been achieved by quantifying quality control results [24].

Some challenges related to the quality of practices, such as DR mammography compared to the indirect CR system from the viewpoints of specificity and sensitivity [43, 46], have been discussed for a long time. The same issues were also observed in our selected studies. Technological changes have an impact on radiographers' practice in mammography, which must be considered in clinical practice and in education. Some issues regarding the standardization of dose reference levels (DRL) implementation and the challenges caused by the variability in image quality assessment were also emphasized (Table 4). This variability may be due to the fact that although there are uniform guidelines for QA and QC [12, 13, 31], a lack of achievement of the recommended standards in European countries has been identified. There are also variations in the recommendations of the available guidelines, emphasizing the importance of harmonizing the quality keys promoting the achievement of a golden standard for continuous quality improvement. It is also important to update these guidelines periodically, introducing the developments observed in
Table 4 Biggest challenges of breast imaging practice according to selected studies

a) Challenges of technical performance

1. cleaning and artefacts: working surfaces, screens, cassettes and processing units

2. AEC: change from manual to AEC, service adjustment to AEC

3. periodic test performance

4. exposure parameters: inappropriate use

5. image receptor: change, damaged or scratched or broken receptors

6. film-screen combination: not spectrally matched or broken

7. viewing conditions

8. breast compression: variations between patients during imaging rounds, between practitioners, imaging sites in association with dose, breast thickness and MGD; use of rigid and flexible compression paddles; different types of practitioner viewpoints and behaviour in using compression force

9. use of positioning sheets

10. implementation of QC programs

b) Challenges of quality of practices

1. positioning

2. image contrast

3. image artefacts

4. variations in image quality in using FS, CR and DR systems

5. use of DRLs: DRLs are defined for standard sized patients which does not always apply to reality

6. implementation of repeat/reject analysis

7. image (FS or DI) processing

8. image labelling and documentation

9. variability in the assessment systems of image quality

c) Challenges of patient centeredness

1. giving seamless and multiprofessional diagnostic services

2. association of heavy workload with deficiencies in patient-centred services: lack of time for the patient

3. promoting breast screening adherence in a radiographer's work

4. patient-centred viewpoint in the use of compression force

technologies and practices based on evidence $[12,13,31]$. Even when radiographers have the knowledge of main guidelines and the strategies to improve practice, their performance cannot be improved if the healthcare organization does not provide all the resources needed (time, materials, training).

Although communication has been mentioned as one of the central issues in the European guidelines [12, 13, 31], according to the analysed studies, it seems this aspect of mammography practice has not been emphasized as much as the issues of technical quality or the quality of practices. The roles among radiology staff can be very different from one country to another [24]. This is an issue also for radiographers. A deficient image quality that may interfere with the diagnosis should be recognized by the radiographer and corrected, if possible. In addition, offering diagnostic services [36], using time to communicate with patients [31], and promoting breastscreening adherence [33] are topics that should be addressed 
more. The result is not the desired for the patient when breast cancer is diagnosed, and the experience can be worse if the patient feels emotionally and mentally neglected or disturbed. The quality of screening can be also improved by efficient interprofessional and intraprofessional collaboration and communication $[13,24,25]$.

\section{Implications}

- Implementation of the European guidelines for quality assurance in breast cancer screening and diagnosis are varying in European countries. All the European countries should reach the same level of observance.

- Undergraduate radiographer training is not sufficient regarding the quality expectations of the mammography screening programmes.

- CPD education in mammography should have equal demands, and it should be mandatory in all the European countries in order to harmonize the education and clinical practice in mammography.

- Development of mammography training and clinical practice should be considered equal to other imaging examinations performed by radiographers, and resourced to reach the highest quality of education and patient services.

- In addition to technical quality and the quality of practice, the patient-centred viewpoint should also be emphasized both in mammography education as well as in clinical practice.

\section{Potential biases and limitations of this integrative review}

This study was performed because the phenomenon in question is not very well known. The use of a blinded review process and a pre-stated search strategy aimed to control biases in this integrative review. The validity of the results is limited by the variability of the imaging standards in breast screening and mammography in the countries where the selected studies of our review had been performed.

The year limit 2010 proved to be relevant, as anticipated, to getting the studies that describe the most contemporary challenges in mammography education and clinical practice. Due to the limited number of the studies that remained after all the review steps, and due to the specific nature of the topic, a previously modified set of ten criteria was applied that is commonly used to evaluate the quality of reporting studies across different methodologies [22, 23]. A benefit of using this checklist is that the evaluation of the studies can be more easily compared across different types of studies, and this evaluation process can be made visible for readers and evaluators. The used criteria revealed poor rankings in the descriptions of study limitations and generalizability as well as the accounts of addressing potential sources of bias. However, this does not exclude the main limitation of integrative reviews, namely the problem of synthesising findings of studies with disparate methodologies.

\section{Conclusions}

The introduction of harmonized guidelines across Europe may serve as an evidence-based tool to be implemented in practice and education. However, the variability in human and material resources as well as in cultural contexts should be considered to improve education and clinical practice. Training in positioning, QC, image quality evaluation, optimization of breast compression, multiprofessional teamwork, client/ radiographer communication, and the patient-centred approach are necessary to meet the challenges of practice identified in the studies analysed in this review.

Acknowledgements The European Commission support for the production of this publication does not constitute an endorsement of the contents which reflects the views only of the authors, and the Commission cannot be held responsible for any use which may be made of the information contained therein.

Open Access This article is distributed under the terms of the Creative Commons Attribution 4.0 International License (http:// creativecommons.org/licenses/by/4.0/), which permits unrestricted use, distribution, and reproduction in any medium, provided you give appropriate credit to the original author(s) and the source, provide a link to the Creative Commons license, and indicate if changes were made.

\section{References}

1. Youlden DR, Cramb SM, Dunn NAM et al (2012) The descriptive epidemiology of female breast cancer: An international comparison of screening, incidence, survival and mortality. Cancer Epidemiol 36:237-248. doi:10.1016/j.canep.2012.02.007

2. Evans A, Whelehan P (2011) Breast screening policy: are we heading in the right direction? Clin Radiol 66:915-9. doi:10.1016/j. crad.2011.03.024

3. Fischer U, Hermann KP, Baum F (2006) Digital mammography: current state and future aspects. Eur Radiol 16:38-44. doi:10.1007 /s00330-005-2848-0

4. Institute of Medicine - National Research Council (2001) Mammography and Beyond: Developing Technologies for the Early Detection of Breast Cancer, 1st edn. National Cancer Policy Board - Institute of Medicine, Washington

5. Ciraj-Bjelac O, Faj D, Stimac D et al (2011) Good reasons to implement quality assurance in nationwide breast cancer screening programs in Croatia and Serbia: results from a pilot study. Eur J Radiol 78:122-128. doi:10.1016/j.ejrad.2009.10.004

6. Committee on New Approaches to Early Detection and Diagnosis of Breast Cancer; National Cancer Policy Board; Board on Science, Technology and EPP and GAD (2005) Saving Women's Lives: Strategies for Improving Breast Cancer Detection and Diagnosis. The National Academies Press, Washington

7. Klabunde C, Bouchard F, Taplin S et al (2001) Quality assurance for screening mammography: an international comparison. $\mathrm{J}$ Epidemiol Community Health 55:204-212 
8. Bassett LW, Hoyt AC, Oshiro T (2010) Digital mammography: clinical image evaluation. Radiol Clin North Am 48:903-15. doi:10.1016/j.rcl.2010.06.006

9. Li Y, Poulos A, Mclean D, Rickard M (2010) A review of methods of clinical image quality evaluation in mammography. Eur J Radiol 74:122-131. doi:10.1016/j.ejrad.2009.04.069

10. Monnin P, Bochud FO, Verdun FR (2010) Using a NPWE model observer to assess suitable image quality for a digital mammography quality assurance programme. Radiat Prot Dosimetry 139:459462. doi:10.1093/rpd/ncq010

11. Marshall G, Punys V, Sykes A (2008) The continuous professional development (CPD) requirements of radiographers in Europe: An initial survey. Radiography 14:332-342. doi:10.1016/j. radi.2006.11.003

12. Reis C, Pascoal A, Sakellaris T, Koutalonis M (2013) Quality assurance and quality control in mammography: a review of available guidance worldwide. Insights Imaging 4:539-553. doi:10.1007 /s13244-013-0269-1

13. European Communities/EUREF (2006) European guidelines for quality assurance in breast cancer screening and diagnosis, 4th edn. European Communities, Luxembourg

14. Radiation and Nuclear Safety Authority (2013) Radiation safety in mammographic examinations.

15. Giordano L, von Karsa L, Tomatis M et al (2012) Mammographic screening programmes in Europe: organization, coverage and participation. J Med Screen 19:72-82. doi:10.1258/jms.2012.012085

16. Peterson EB, Ostroff JS, DuHamel KN et al (2016) Impact of provider-patient communication on cancer screening adherence: A systematic review. Prev Med (Baltim). doi:10.1016/j. ypmed.2016.09.034

17. Fallis A (2011) Joanna Briggs Institute Reviewers' Manual. doi: 10.1017/CBO9781107415324.004

18. Whittemore R, Knafl K (2005) The integrative review: updated methodology. J Adv Nurs 52:546-553. doi:10.1111/j.13652648.2005.03621.x

19. Joanna Briggs Institute (2014) New JBI Levels of Evidence. Adelaide

20. Strobe Group (2007) STROBE Statement - checklist of items that should be included in reports of observational studies.

21. Hafslund B, Wammen NM (2009) Mammography screening from the perspective of quality of life: a review of the literature. Scand $\mathrm{J}$ Caring Sci 23:539-548. doi:10.1111/j.1471-6712.2008.00634.x

22. Metsälä E, Henner A, Ekholm M (2014) Quality assurance in digital dental imaging: a systematic review. Acta Odontol Scand 72: 362-371. doi:10.3109/00016357.2013.840736

23. Metsälä E, Vaherkoski U (2014) Medication errors in elderly acute care - a systematic review. Scand J Caring Sci 28:12-28. doi:10.1111/scs.12034

24. Ciraj-Bjelac O, Avramova-Cholakova S, Beganovic A, et al. (2011) Image quality and dose in mammography in 17 countries in Africa, Asia and Eastern Europe: Results from IAEA projects. Eur J Radiol Article in:1-8. doi: 10.1016/j.ejrad.2011.05.026

25. Brnić Z, Blašković D, Klasić B, et al. (2011) Image quality of mammography in Croatian nationwide screening program: Comparison between various types of facilities. Eur J Radiol Article in:1-8. doi: 10.1016/j.ejrad.2011.06.020

26. Gurdemir B, Aribal E (2012) Assessment of mammography image quality in Istanbul city. Diagnostic Interv Radiol 18:468-472. doi:10.4261/1305-3825.DIR.5400-11.1

27. O'Leary D, Rainford L (2013) A comparison of mean glandular dose diagnostic reference levels within the all-digital Irish national breast screening programme and the Irish symptomatic breast services. Radiat Prot Dosimetry 153:300-308. doi:10.1093 $/ \mathrm{rpd} / \mathrm{ncs} 112$

28. Mercer CE, Szczepura K, Kelly J et al (2015) A 6-year study of mammographic compression force: Practitioner variability within and between screening sites. Radiography 21:68-73. doi:10.1016/j. radi.2014.07.004

29. Branderhorst W, de Groot JE, Highnam R et al (2015) Mammographic compression - A need for mechanical standardization. Eur J Radiol 84:596-602. doi:10.1016/j.ejrad.2014.12.012

30. Murphy F, Nightingale J, Hogg P et al (2015) Compression force behaviours: An exploration of the beliefs and values influencing the application of breast compression during screening mammography. Radiography 21:30-35. doi:10.1016/j.radi.2014.05.009

31. Reis C (2013) Digital Mammography: Characterisation Of Practice And Equipment Performance In Portuguese Healthcare Providers. Universidade Católica Portuguesa

32. Boyce M, Gullien R, Parashar D, Taylor K (2015) Comparing the use and interpretation of PGMI scoring to assess the technical quality of screening mammograms in the UK and Norway. Radiography 21:342-347. doi:10.1016/j.radi.2015.05.006

33. Burgess $\mathrm{C}$, Teasdale E, Omar L et al (2012) Training radiographers to deliver an intervention to promote early presentation of breast cancer. Radiography 18:232-237. doi:10.1016/j.radi.2012.06.003

34. Moreira IC, Ventura SR, Ramos I et al (2015) Development and Assessment of an E-Learning Course on Breast Imaging for Radiographers: A Stratified Randomized Controlled Trial. J Med Internet Res 17:e3. doi:10.2196/jmir.3344

35. Timmers J, ten Voorde M, Engen RE et al (2015) Mammography with and without radiolucent positioning sheets: Comparison of projected breast area, pain experience, radiation dose and technical image quality. Eur J Radiol 84:1903-1909. doi:10.1016/j.ejrad.2015.07.005

36. Mathers SA, McKenzie GA, Robertson EM (2013) "It was daunting": Experience of women with a diagnosis of breast cancer attending for breast imaging. Radiography 19:156-163. doi:10.1016/j.radi.2012.11.004

37. Mercer CE, Hogg P, Szczepura K, Denton ERE (2013) Practitioner compression force variation in mammography: A 6-year study. Radiography 19:200-206. doi:10.1016/j.radi.2013.06.001

38. Broeders MJM, ten Voorde M, Veldkamp WJH et al (2015) Comparison of a flexible versus a rigid breast compression paddle: pain experience, projected breast area, radiation dose and technical image quality. Eur Radiol 25:821-829. doi:10.1007/s00330-014-3422-4

39. Leal J, Andrade AS, Ribeiro R (2012) Continuous Professional Development : The perspective of radiographers in private and public institutions of Lisbon region. Eur Soc Radiol C-1815:1-17. doi:10.1594/ecr2012/C-1815

40. Moreira IC, Amaral I, Domingues I et al (2012) INbreast Toward a Full-field Digital Mammographic Database. Acad Radiol 19:236248. doi:10.1016/j.acra.2011.09.014

41. Pisano ED, Gatsonis C, Hendrick E et al (2005) Diagnostic performance of digital versus film mammography for breast-cancer screening. N Engl J Med 353:1773-1783. doi:10.1056/NEJMoa052911

42. Pisano ED, Hendrick RE, Yaffe MJ et al (2008) Diagnostic accuracy of digital versus film mammography: exploratory analysis of selected population subgroups in DMIST. Radiology 246:376-83. doi:10.1148/radiol.2461070200

43. Chiarelli AM, Edwards SA, Prummel MV et al (2013) Digital Compared with Screen-Film Mammography: Performance Measures in Concurrent Cohorts within an Organized Breast Screening Program. Radiology 268:684-693. doi:10.1148/radiol.13122567

44. Whelehan P, Evans A, Wells M, MacGillivray S (2013) The effect of mammography pain on repeat participation in breast cancer screening: A systematic review. Breast 22:389-394. doi:10.1016 /j.breast.2013.03.003

45. Zhou Y, Scott A, Allahverdian J, Frankel S (2014) Evaluation of automatic exposure control options in digital mammography. J Xray Sci Technol 22:377-394. doi:10.3233/XST-140433

46. Alsleem HUP, Mong KS, Davidson R (2014) Effects of radiographic techniques on the low-contrast detail detectability performance of digital radiography systems. Radiol Technol 85:614-622 